\title{
Decidability of epimorphisms of dimension groups and certain modules $\dagger$
}

\author{
K. H. KIM ANDF. W. ROUSH \\ Mathematics Research Group, Alabama State University, Montgomery, \\ Alabama 36195, USA
}

(Received 22 January 1988 and revised 27 April 1988)

\begin{abstract}
We give an algorithm for deciding the existence of epimorphisms of finitely additively generated $Z[t]$ modules and dimension groups. This shows that the existence of eventual right resolving shift factor maps for mixing finite type shifts is decidable.
\end{abstract}

\section{Introduction}

In [8], it was established that shift equivalence is decidable for matrices over $Z^{+}$ (nonnegative integers) where elements $a$ and $b$ of a semigroup are shift equivalent provided that there exist semigroup elements $r$ and $s$ and $n \in Z^{+}$such that $r a=b r$, $a s=s b, r s=b^{n}, s r=a^{n}$. This work made use in turn of [7], by reducing shift equivalence over $Z^{+}$to shift equivalence over $Z$ and also made use of [5], [6] to find finite sets of generators for certain matrix groups and their cosets.

Shift equivalence is essentially the existence of an isomorphism between dimension groups, where the dimension group associated with a linear transformation $\tau$ of an abelian group $G$ is the direct limit $\hat{G}$ of $G$ under $\tau$ as a $Z[\tau]$-module.

Definition. For matrices $A, B$, of sizes $m_{0} \times m_{0}, n_{0} \times n_{0}$ we define modules $\mathscr{M}_{A}, M_{B}$ to be $Z^{m_{0}}, Z^{n_{0}}$ with $\tau$ acting on the right by $A, B$ respectively.

We define dimension groups $\mathfrak{D}_{A}, \mathfrak{D}_{B}$ to abstractly be the direct limits of $\mathscr{M}_{A}, \mathcal{M}_{B}$ under the $\tau$ action, i.e., the quotients of

$$
\bigoplus_{1}^{\infty} \mathcal{M}_{A}, \quad \bigoplus_{1}^{\infty} \mathcal{M}_{B}
$$

by the relations $(0, \ldots, 0, x, 0, \ldots, 0) \sim(0, \ldots, 0, \tau x, 0, \ldots, 0)$ whenever $x, \tau x$ appear in coordinates $(i, i+1)$ for some $i \in Z^{+}$.

As subgroups of $\mathscr{M}_{A} \otimes Q$ and $\mathscr{M}_{B} \otimes Q$ we define $\mathfrak{D}_{A}, \mathfrak{D}_{B}$ to be

$$
\bigcup_{i \in Z^{+}}\left(Z^{m_{0}}\right) A_{d}^{i} \bigcup_{i \in Z}\left(Z^{n_{0}}\right) B_{d}^{i}
$$


where $A_{d}, B_{d}$ are Drazin inverses of $A, B$, i.e., $A_{d} A=A A_{d}, B_{d} B=B B_{d}, A_{d} A^{n+1}=A^{n}$, $B_{d} B^{n+1}=B^{n}$ for some $n \in Z^{+}, \operatorname{Rank}\left(A_{d}\right)=\operatorname{Rank}\left(A^{n}\right), \operatorname{Rank}\left(B_{d}\right)=\operatorname{Rank}\left(B^{n}\right)$.

Equivalently one can say [1] that the dimension group $\mathfrak{D}_{A}$ is the set of all vectors $v \in Q^{m_{0}}$ in the image of all large powers of $A$ such that $v A^{r} \in Z^{m_{0}}$ for some $r \in Z^{+}$.

In [9], we showed that the existence of epimorphisms of finitely additively generated modules over finitely additively generated commutative rings in general is undecidable.

In [5] it is proved that among much else the existence of the isomorphism of such modules is decidable. Here we prove that an algorithm exists to construct epimorphisms of modules over the ring $Z[t]$ and that the same procedure serves to decide the existence of epimorphisms of dimension groups. This extends the isomorphism result of [4].

Definition. The subshift of finite type associated with an $n$-square $(0,1)$-matrix $M$ is the subset of $\underline{N}^{z}=\left\{\left(\ldots, a_{-2}, a_{-1}, a_{0}, a_{1}, a_{2}, \ldots\right): a_{i} \in N\right\}, N=\{1,2, \ldots, n\}$ such that for all $i$, the $a_{i}, a_{i+1}$ entry of $M$ is 1 . It is topologized by the product topology of $N^{Z}, N$ discrete. The shift map sends $\left(x_{i}\right)$ to $\left(y_{i}\right), y_{i}=x_{i+1}$ for $i \in Z$.

Definition. A Shift map from one subshift to another is a continuous map commuting with the shift.

Definition. A factor map is continuous onto shift map. That it is right closing means if $x_{i}=y_{i}$ for all $n<n^{\prime}$ for some $n^{\prime} \in Z$ and $f(x)=f(y)$, then $x=y$.

Boyle et al. [1] proved that the existence of right closing factor maps for some power of a subshift is equivalent to the existence of an epimorphism of dimension groups provided the entropies are equal and the matrices are primitive.

The entropy of a shift is the logarithm of the largest eigenvalue of a matrix. A matrix $A$ is primitive if $A^{n}>0$ for some $n \in Z^{+}$. The epimorphism condition on dimension groups can be restated as existence of integer matrices $R, S$ and an integer $n \in Z^{+}$such that

$$
A S=S B, \quad R S=B^{n} .
$$

(An epimorphism of modules says $R S=I$. Here $I$ is an identity matrix.)

We work with arbitrary matrices $A, B$, over $Z$. But the topological applications are only for primitive nonnegative $A, B$. Our method for treating this problem is to split it into separate problems at the different eigenvalues of $B$. Then we can reduce to the nilpotent case.

The structure of nilpotent modules $\mathcal{M}$ over $\Omega[t]$ where $\Omega$ is an algebraic number ring is rationally determined by the filtered abelian group $\mathcal{M} / t \mathcal{M}$ filtered by its intersection with $\operatorname{Ker}\left(t^{n}\right)$. Thus existence of congruence classes of near epimorphisms (maps with bounded cokernel) on $\mathcal{M}$ reduces to the same problem for the filtered $\Omega$-module $\mathcal{M} / t \mathcal{M}$, and in fact to one for epimorphisms. For existence of epimorphisms which modulo an ideal $I$ are given of finitely generated torsion free filtered modules $\mathscr{M}$ to $\mathcal{N}$ such that the filteration subgroups are pure (not divisible) we find an explicit criterion. This is that

$$
\operatorname{Rank}\left(\mathscr{M} / \mathscr{M}_{i}\right) \geq \operatorname{Rank}\left(\mathcal{N} / \mathcal{N}_{i}\right)
$$


and if equality holds

$$
\mathscr{M} / \mathcal{M}_{i} \simeq \mathcal{N} / \mathcal{N}_{i}
$$

and that $g$ from $\mathcal{M} / \mathcal{M}_{i}$ to $\mathcal{N} / \mathcal{N}_{i}$ has as a determinant the modulo $\mathscr{I}$ reduction of a unit. The construction splits $\mathcal{M}$ into a direct sum of ideals and constructs a lifting of $g$ on each in turn.

We avoid the division algebra problems of [8] by our primitivity assumption.

They occur only shift equivalence of nonnegative nonprimitive matrices. That requires a little more than dimension group isomorphism. Sometimes we use the terms integrally, rationally to indicate whether fractions occur.

Definition. A homomorphism $f$ from $\mathcal{M}$ to $\mathcal{N}$ of modules over a ring $\Omega$ exists rationally (integrally) means $f$ exists over from $\mathcal{M} \otimes Q$ to $\mathcal{N} \otimes Q$ (from $\mu$ to $\mathcal{N}$ ).

Basic constructions giving effective computability for linear algebra and field theory can be found in [2], [4]. We need to be able to solve linear equations and congruences in algebraic number rings and fields, compute polynomials, find integral and rational bases, construct fields based on specific eigenvalues, determine Galois group action on specific elements, factor ideals in algebraic A0, A2, A6, A9, A10, A11, A14, A15, of [4] cover these. We give additional algorithms for rings $\Omega[1 / \omega]$ in $\S 3$.

\section{Reduction to a single eigenvalue}

Considering matrices $A$ and $B$, for each eigenvalue $\omega$ of $A$ there exists a polynomial $e_{\omega}[t]$ defining a projection on the eigenspace of $\omega$ and vanishing on all other eigenspaces of $A, B$. If an epimorphism $\mathfrak{D}_{A}$ to $\mathfrak{D}_{B}$ or $\mathcal{M}_{A}$ to $\mathcal{M}_{B}$ exists rationally, the eigenvalues of $B$ must be a subset of those of $A$. To find $e_{\omega}$, it suffices to have

$$
e_{\omega}(t)^{2}-e_{\omega}(t) \equiv 0(\bmod c(t)),
$$

where $c$ is the characteristic polynomial of $A, e_{\omega}(\omega) \neq 0, e_{\omega}\left(\omega_{i}\right)=0$ for all eigenvalues $\omega_{i} \neq \omega$ of $A$. We can find $\epsilon_{\omega}$ by taking a polynomial

$$
\frac{c(t)}{(t-\omega)^{s}}
$$

and multiplying by an inverse in the ring of polynomials modulo $(t-\omega)^{s}$.

We use finite systems of congruences on a set $X$ understood as contained in a finitely generated module $\mathcal{M}$ over some integral domain $\mathfrak{J}_{0}$. This means a finite module $\mathscr{M}_{1}$ over $X$, a module homomorphism $\mathscr{M} \rightarrow \mathscr{M}_{1}$ specified in terms of generators and a subset of $\mathcal{M}_{1}$ understood as the image of $X$. These congruences are said to involve a prime ideal $\mathscr{P}$ of $\Im_{0}$ if $\mathscr{P} \mathscr{M}_{1} \neq \mathscr{M}_{1}$. We also allow finite systems of congruences on a product of such sets $X_{1} \times \cdots \times X_{n}$. By this we mean a system for each $X_{i}$ as above which is a subset $Y_{i}$ of a module $\mu_{1}(i)$ together with a subset of $Y_{1} \times \cdots \times Y_{n}$.

On a finitely generated module $\mathcal{M}$, a property depends on a finite system of congruences if for some element $m \in \mathfrak{I}_{0}$, the property is invariant under addition of an element of $m \mathcal{M}$ and $\Im_{0} / m \Im_{0}$ is finite. Let $R, S$, give an epimorphism of dimension groups from $A$ to $B$. Let $A_{\omega}=e_{\omega}(A) A, B_{\omega}=e_{\omega}(B) B, R_{\omega}=e_{\omega}(B) \operatorname{Re}_{\omega}(A)$, $S_{\omega}=e_{\omega}(A) S e_{\omega}(B)$. Then $A=\sum A_{\omega}, B=\sum B_{\omega}$, since $\sum e_{\omega}$ is the identity. Also $\sum S_{\omega}=\sum e_{\omega}(A) S=S$. However $\sum R_{\omega} \neq R$ in general. 
Let $Q(\omega)$ be the algebraic number field generated by the eigenvalue $\omega$ over the rationals. Let $\Omega_{\omega}$ denote the ring of algebraic integers of $Q(\omega)$.

THEOREM 2.1. Let $\boldsymbol{K}$ be the field generated over $Q$ by all eigenvalues of a matrix $B$. Assume that the minimal polynomial of $B$ divides that of $A$. Let $S$ be an $m_{0} \times n_{0}$ matrix over $K$ where $A$ and $B$ are $m_{0} \times m_{0}$ and $n_{0} \times n_{0}$.

Rationally $S$ gives an epimorphism of dimension groups from $\mathfrak{D}_{A}$ to $\mathfrak{D}_{B}\left(\mathcal{M}_{A}\right.$ to $\mathcal{M}_{B}$ ) if and only if $S_{\omega}=e_{\omega}(A) S$ does so for all eigenvalues $\omega$ of $B$. The matrix $S$ is rational over $Q$ if and only if $S_{\omega}$ are conjugate for conjugate eigenvalues $\omega$ and in $Q(\omega)$. The condition that $S$ be integral and that some integral $R$ exist can be stated as (i) a finite system of congruences on the $S_{\omega}$ only over $\Omega_{\omega}$; (ii) a bound on the denominators of $S_{\omega}$; (iii) $S_{\omega}$ are epimorphisms except at a finite given set of primes of $\Omega_{\omega}$. In the dimension group case $S_{\omega}$ will be a matrix over $\Omega_{\omega}(1 / \omega)$ and congruences will be to moduli not dividing $\omega$, the bound will be on the factors of denominators relatively prime to $\omega$.

Proof. From $A S=S B$, we have

$$
\begin{aligned}
e_{\omega}(A) S & =S e_{\omega}(B)=S_{\omega}, \\
A_{\omega} S_{\omega} & =A_{\omega} e_{\omega}(A) S=A e_{\omega}(A) S=A S e_{\omega}(B)=S B e_{\omega}(B)=S_{\omega} B_{\omega}, \\
R_{\omega} S_{\omega} & =e_{\omega}(B) R e_{\omega}(A) S e_{\omega}(B)=e_{\omega}(B) R S e_{\omega}(B)^{2}, \\
& =e_{\omega}(B) B^{n} e_{\omega}(B)^{2}=B_{\omega}^{n},
\end{aligned}
$$

if $R_{\omega}, S_{\omega}$ are defined as above so if $S$ gives an epimorphism of dimension groups so does each $S_{\omega}$. Conversely, let each $S_{\omega}$ give an epimorphism of dimension groups rationally, and $S_{\omega}=e_{\omega}(A) S_{\omega} e_{\omega}(B)$. We can define $R_{\omega}$ by the existence of an epimorphism, such that $R_{\omega}=e_{\omega}(B) R_{\omega} e_{\omega}(A)$. Then

$$
\begin{aligned}
& \sum A_{\omega} \sum S_{\omega}=\sum A_{\omega} S_{\omega}=\sum S_{\omega} B_{\omega}=\sum S_{\omega} \sum B_{\omega}, \\
& \sum R_{\omega} \sum S_{\omega}=\sum R_{\omega} S_{\omega}=\sum B_{\omega}^{n}=\left(\sum B_{\omega}\right)^{n} .
\end{aligned}
$$

So $\sum S_{\omega}$ defines an epimorphism of dimension groups. If $S$ is rational over $Q$ then $e_{\omega}(A)$ are conjugate for conjugate $\omega$ and so are $S_{\omega}$. Conversely, if $S_{\omega}$ are conjugate for conjugate $\omega$ then $\sum S_{\omega}$ is invariant under the Galois group entrywise. If $S$ is integral then $S_{\omega}$ will have denominators arising solely from the fixed matrices $e_{\omega}(A)$. The condition $\sum S_{\omega}$ is integral given that this bound on the denominators amounts to a finite system of congruences and that each $S_{\omega}$ modulo integral matrices belongs to a congruence class such that the sum of those congruence classes is integral.

In the dimension group case, for $A^{n} \sum S_{\omega}$ to be integral for sufficiently large $n$ we may as well assume each $S_{\omega}$ is integral at primes dividing $\omega$, since for $n$ large $A^{n} S_{\omega}$ has this property. Then at any prime $\pi$ we have that $\sum_{\pi \nmid \omega} S_{\omega}$ is integral which is equivalent to a finite system of congruences.

The matrix $S$ gives the mapping, existence of $R$ says that it is an epimorphism on dimension groups, more precisely that the image of $S$ on integer vectors contains the image of $A^{n}$ for some $n$. This property can be localized. Let $G_{1}, G_{2}$ be the dimension groups (contained in some $Q^{n}$ ) of $A, B$.

Let $\mathfrak{F}$ be any algebraic number field and $\Omega_{\pi}$ the localization of its ring of algebraic integers at any prime $\pi$. Consider $S$ as a mapping from a group $G_{1}$ to a group $G_{2}$. 
Then the cokernel of $S$ over $\Omega_{\pi}$ is

$$
G_{2} \otimes \Omega_{\pi} / S\left(G_{1}\right) \otimes \Omega_{\pi} \simeq G_{2} / S\left(G_{1}\right) \otimes \Omega_{\pi}
$$

by right exactness of tensor products of abelian groups [2, Proposition 2.4.5]. Suppose $S$ is not epic. If the cokernel is nontorsion then for all $\pi$, (by [2, Proposition 7.2.1]), $G_{2} / S\left(G_{1}\right) \otimes \Omega_{\pi}$ is nonzero. If the cokernel is torsion and has a nonzero $p$ primary part $C_{p}, C_{p}$ is then a summand and for all $\pi$ dividing $p, C_{p} \otimes \Omega_{\pi}$ is direct limit of $F \otimes \Omega_{\pi}$ where $F$ are the finite subgroups of $C_{p}$, under inclusion. Each $F \otimes \Omega_{\pi}$ is nonzero and we have monomorphisms on them induced by inclusion. So $C_{p} \otimes \Omega_{\pi} \neq\{0\}$. So it suffices that $S$ be an epimorphism locally at all $\pi$, in fact one $\pi$ from each conjugacy class since conjugate $\pi$ divide the same $p$. Here we take primes $\pi$ in the field $\boldsymbol{K}$.

If $R, S$ exist then $S_{\omega}$ must be an epimorphism at all primes not dividing $\omega$ or any denominator of $e_{\omega}$ because of the existence of $R_{\omega}$, and the fact $B_{\omega}$ is invertible on the dimension group at these primes. At all other primes not dividing $\omega$ we must have a bound on the order of the cokernel (at all primes in the module case) from the bound on the denominators of $R_{\omega}$. As $\Omega_{\pi}$-modules the dimension groups will have finite rank and bounded denominators in $\pi$.

The global dimension groups are not finitely generated as modules over any subring of $\Omega \otimes Q$ in general. However, we can explicitly find a spanning set for the local dimension groups

$$
\bigcup_{i}\left(\Omega_{\pi}\right)^{m_{0}} A_{\omega}^{-i}
$$

at each prime $\pi$ not dividing $\omega$. The matrices $A_{\omega}^{-i}$ exist over the complete ring $\Omega_{\pi}$ since $\pi$ does not divide Det $\left(A_{\omega}\right)$. So we can find a finite spanning set, in fact $I$, $A_{\omega}, \ldots, A_{\omega}^{m_{0}-1}$ span. Moreover, we can explicitly find the congruences such that

$$
\sum_{\omega} v_{\omega}
$$

over $\pi$ not dividing $\omega$ is $\pi$ integral, in terms of such a spanning set.

At primes not dividing any $\omega$ or denominator of $e_{\omega}$, integrality of $S_{\omega}$ and epimorphism of $S_{\omega}$ implies those properties for $S$ since we have a direct sum splitting of the dimension groups by $e_{\omega}$. In the case of finitely generated modules at the finitely many other $\pi$, we have bounds on denominators and sizes of cokernels. $S$ will be a epimorphism at $\pi$ if and only if it is an epimorphism modulo some $\pi^{n}(\bmod \pi$ into the dimension group) which is a finite system of congruences. Next we give details.

In the dimension group, the matrices $e_{\omega}(B)$ represent the dimension group for $B$ as a subset of a direct sum over $\omega$. This subset is specified by $\sum v_{\omega} B_{\omega}^{n}$ being integral for sufficiently large $n$, where $v_{\omega}=v e_{\omega}(B)$ for $v$ in the dimension group. For conjugate $\omega$, the $v_{\omega}$ must be conjugate, so we work with 1 representative for each conjugacy class. Let $\chi=\{\omega: \pi$ does not divide $\omega\}$. The term $v_{\omega} B_{\omega}^{n}$ will always be integral for large $n$, at $\pi \mid \omega$ since $B_{\omega}$ to some power is divisible by $\pi$. Integrability of

$$
\sum_{\chi} v_{\omega} B_{\omega}^{n}
$$

for $n$ large since denominators are bounded, can be stated in terms of finite congruences on these $v_{\omega}$, whose modulus is a power of $\pi$. 
The matrix $\sum S_{\omega}$ will be then epic locally at $\pi$ if and only if it is rationally epic and $\sum_{\chi} S_{\omega}$ is epic since any rational vector $v_{\omega}, w \notin \chi$ is in the image. Then $\sum_{x} S_{\omega}$ will be epic if and only if it is epic modulo $\pi$ into this portion of the dimension group locally at $\pi$. This condition is equivalent to a finite system of congruences.

COROlLARY. To decide if an epimorphism of $Z[t]$-modules or dimension groups exists it suffices to be able to decide for all algebraic number rings $\Omega_{0}$, all nonzero $\omega \in \Omega_{0}$, all finite sets $\chi_{0}$ of primes of $\Omega$ and all nilpotent finitely generated $\Omega_{0}[1 / \omega, t]$ modules $\mathcal{M}_{1}, \mathcal{M}_{2}$ whether a module homomorphism $\mathcal{M}_{1} \rightarrow \mathcal{M}_{2}$ exists which is an epimorphism at all primes of the complement $\tilde{\chi}_{0}$ of $\therefore$ and satisfies a finite set of congruences in primes not in $\tilde{\chi}_{0}$ nor dividing $\omega$. (For modules $\omega=1$.)

Proof. In the dimension group case, the modules $\mathcal{M}_{1}, \mathcal{M}_{2}$ are $D_{1} e_{\omega}(A), D_{2} e_{\omega}(B)$ where $D_{1}, D_{2}$ are the two dimension groups, taken over $\Omega_{\omega}[1 / \omega]$. They are acted on by $\left(A_{\omega}-\omega I\right),\left(B_{\omega}-\omega I\right)$ which are nilpotent. They are finitely generated since if $m_{0}$ is the dimension of $A$

$$
D_{1} e_{\omega}(A)=\bigcup_{i}\left(\Omega_{\omega}[1 / \omega]^{m_{0}}\right) A_{\omega}^{-i}
$$

but we can bound all denominators in $A_{\omega}^{-i}$ at primes not in $\omega$, independently of $i$. The condition that $S_{\omega}$ map $\mu_{1}$ into $\mathcal{M}_{2}$ bounds all denominators not dividing $\omega$. The condition that $\sum e_{\omega}(A)^{n} S_{\omega}$ for large $n$ be integral amounts to a finite set of additional congruences as in the previous proof. And the condition that the sum be an epimorphism at the finite set of primes $\pi$ not dividing $\omega$ but occurring in denominators of $e_{\omega}$ is another finite set of congruences. For the module case, these hold except that we have only bounded denominators at primes dividing $\omega$.

Definition. A near epimorphism of $\Omega_{0}[1 / \omega, t]$-modules (dimension groups) is an epimorphism except at a given specified set of primes provided with a bound on the cokernel at those primes (exclusive of $\omega$ in the dimension group case).

So it suffices to decide the congruence classes of near epimorphisms.

\section{Properties of $\Omega$-modules}

Let $\Omega=\Omega_{0}[1 / \omega]$ where $\Omega_{0}$ is an algebraic number ring, nonzero $\omega \in \Omega_{0}$. The following results can be found in Serre [10]. From here on modules are left modules.

Definition. A pure submodule $\mathcal{N}$ of $\mathcal{M}$ is one such that $\mathscr{M} / \mathcal{N}$ is torsion free.

THEOREM 3.1. Let $\Omega$ be $\Omega_{0}[1 / \omega]$ for an algebraic number ring $\Omega_{0}$, nonzero $\omega \in \Omega_{0}$. Then (1) torsion free finitely generated $\Omega$-modules are projective; (2) a pure submodule is a summand; (3) every such module is a direct sum of ideals; and (4) $\mathscr{I}_{1} \otimes \mathscr{I}_{2} \simeq \mathscr{I}_{1} \mathscr{I}_{2} \oplus \Omega$ for any two ideals $\mathscr{I}_{1}, \mathscr{I}_{2}$ of $\Omega$.

THEOREM 3.2. If $\mathcal{M}_{1}, \mathcal{M}_{2}, \mathscr{M}_{3}$ are finitely generated torsion free $\Omega$-modules and $\mathcal{M}_{1} \oplus \mathcal{M}_{3} \simeq \mathcal{M}_{2} \oplus \mathcal{M}_{3}$ then $\mathcal{M}_{1} \simeq \mathcal{M}_{2}$.

We need various background algorithms for working in rings $\Omega_{0}[1 / \omega]=\Omega$. We 
suppose modules finitely generated and given by generators and relations; module homomorphisms given by the image of some generating set.

Algorithm 1 . Given a module $\mathcal{M}$ over $\Omega$ we can find a module $\mathcal{M}_{0}$ over $\Omega_{0}$ such that $\mathcal{M}_{0} \otimes \Omega[1 / \omega]=\mathcal{M}$. We just take the same generators and relations as for $\mathcal{M}$, but multiply relations by a power of $\omega_{0}$ until they are integral.

Algorithm 2. Given a module $\mathcal{M}$ over $\Omega$ we can find its torsion submodule. We can construct $\mathscr{M}_{0} \otimes Q$ using linear equations and embed it in $Q^{n}$. Then we construct the map $\mathcal{M}_{0} \rightarrow \mathcal{M}_{0} \otimes Q$. Choose a free module $\mathscr{F}_{0}$ whose basis is in 1-1 correspondence with generators of $\mathcal{M}_{0}$. Find generators of the kernel $K_{0}$ of $\mathscr{F}_{0} \rightarrow \mathcal{M}_{0} \rightarrow \mathcal{M}_{0} \otimes Q$ by expressing the map as a matrix $B$ over $Z$ and writing $B$ as $B_{0} D_{0} B_{1}$ where $B_{i}$ are unimodular, $D_{0}$ diagonal. Let $R_{0}$ be the subgroup of relations. Write out generators for $\boldsymbol{R}_{\mathbf{0}}$ as $\boldsymbol{Z}$-module, multiplying the given relations by an additive basis for $\Omega_{0}$ over $Z$. Now we find generators for $R_{0} \cap K_{0}$ as abelian group. These can be found by linear algebra over $\boldsymbol{Z}$.

Algorithm 3. Given a torsion module $\mathcal{M}$ we can compute its order and express it as a direc: sum $\Omega / \mathscr{I}_{j} \Omega$ for ideal $\mathscr{I}_{j}$. We obtain $\mathcal{M}_{0}$. We compute its order by obtaining generators and relations over $\boldsymbol{Z}$ and its module structure. Then we try all possibilities for its structure in terms of the finite number of ideals $\mathscr{I}_{j}$ whose norms divide the order. Let $\mathscr{I}_{j}$ be powers of primes. To pass back to $\mathcal{M}$ we divide out all summands $\mathscr{I}_{j}$ is a power of a prime dividing $\omega$.

Algorithm 4. Given a homomorphism $\mathscr{M} \rightarrow \mathcal{N}$ we can determine its kernel, image, and cokernel. To do this we pass to $\mathcal{M}_{0}$ over $\Omega_{0}$ then solve the problem over $\boldsymbol{Z}$.

Algorithm 5. We can determine the rank of a module. This is linear algebra over $\boldsymbol{Q}$. Algorithm 6. Given a homomorphism from $\mathscr{M}$ to a finite module $\mathscr{T}$ (i.e., congruences) we can determine if any element of $\mathscr{T}$ lifts to $\mathcal{M}$ and if so find an element of its inverse image. It suffices to solve this problem for $\mathcal{M}_{0}$. But that reduces to a linear problem over $\boldsymbol{Z}$.

Algorithm 7. Given $\mathscr{P}$ projective and an epimorphism $\mathscr{M} \rightarrow \mathscr{P}$ we can determine a splitting $\mathscr{M} \simeq \mathscr{P} \oplus K$. To do this we compute the finitely generated $\Omega$-modules $\operatorname{Hom}(\mathscr{P}, \mathscr{M}) \operatorname{Hom}(\mathscr{P}, \mathscr{P})$ and the induced mapping, and use Algorithm 6 lift the identity map $\mathscr{P}$ to $\mathscr{P}$.

Algorithm 8. Given a pure submodule $\mathscr{P}$ of $\mathcal{M}$ we can express $\mathcal{M}=\boldsymbol{K} \oplus \mathscr{P}$. We follow Algorithm 7 but use $\operatorname{Hom}(\mathscr{M}, \mathscr{P})$, Hom $(\mathscr{P}, \mathscr{P})$. These means (2) constructive use A13 of [4] to make $\mathscr{I}_{1} \cap \Omega_{0}, \mathscr{I}_{2} \cap \Omega_{0}$ relatively prime and apply Algorithm 7 to the map $\mathscr{I}_{1} \oplus \mathscr{I}_{2} \rightarrow \Omega$ sending $(x, y)$ to $x+y$. Here $\mathscr{I}_{j}=\left(\mathscr{I}_{j} \cap \Omega_{0}\right) \otimes \Omega$ since if $y \in \mathscr{I}_{j}$ the $y \omega^{n} \in \mathscr{I}_{j} \cap \Omega_{0}$ for $n$ sufficiently large. A finitely generated $\Omega$-module $\mathcal{M}$ can be uniquely expressed as a direct sum of copies of $\Omega$ and a single ideal whose class is unique by Theorems 3.1, 3.2.

Algorithm 9. We can express any torsion free module $\mathcal{M}$ as a direct sum of copies of $\Omega$ and a single ideal $\mathcal{N}$, if $\mathcal{M}$ is nonzero. To do this, take a single generator $x \in \mathcal{M}$. Let $\mathcal{N}$ be the submodule spanned by $\left\{x / a: a \in \Omega_{0}, x / a \in \mathcal{M}_{0}\right\}$. There are only a finite number of possibilities for $a$, apart from units, considering the torsion subgroup of 
$M / \Omega x$. By A12, A10 of [4] we can write out each $a$ and test $x / a$ over $Z$ to be in $\mathscr{M}_{0}$. Then $\mathcal{N}$ is a rank 1 pure submodule, therefore a summand. Any rank 1 torsion free module can be mapped into $\Omega \otimes Q$ monomorphically and then into $\Omega$. Now use (4) to adjust the summands.

Algorithm 10. We can determine the ideal class group of $\Omega$ and the class of any ideal. For $\Omega_{0}$, this is A10, A11, A12 of [4]. The class of an ideal $\mathscr{I}$ is the image of the class of $\mathscr{I} \cap \Omega_{0}$. An ideal $\mathscr{I}_{0}$ in $\Omega_{0}$ is principal in $\mathscr{I}$ if and only if its class is a product of classes of primes dividing $\omega$. All primes dividing $\omega$ become $\Omega$. Conversely if $\mathscr{I}_{0} \Omega=\Omega$ then $\omega^{n} \in \mathscr{I}_{0}$ and $\mathscr{I}_{0}$ involves only primes of $\omega$.

An exact duality exists for torsion free finitely generated $\Omega$-modules sending $\boldsymbol{M}$ to $\operatorname{Hom}_{\Omega}(\mathscr{M}, \Omega)$. A map is a monomorphism with pure image if and only if it has a left inverse if and only if its dual is an epimorphism.

Proposition 3.3. For $\mathcal{M}, \mathcal{N}$ torsion free $\Omega-$ modules and a nonzero ideal $\mathscr{I}$ of $\Omega$ any homomorphism

$$
\bar{f}: \mathscr{M} / \mathscr{I} \mathscr{M} \rightarrow \mathcal{N} / \mathscr{I N}
$$

lifts to an $\Omega$-homormorphism $f: \mathcal{M} \rightarrow \mathcal{N}$.

Proof. It suffices to check this for two icieal classes. But realize them by ideals whose norms are relatively prime to $\mathscr{I}$ by Algorithm 13 of [4] then modulo $\mathscr{I}$ both are isomorphic to $\Omega$. Take a map $\Omega$ to $\Omega$ realizing the map modulo $\mathscr{I}$. Compose with maps $\mathscr{M}$ to $\Omega, \Omega$ to $\mathcal{N}$ which are isomorphisms modulo $\mathscr{I}$.

Proposition 3.4. Let $\mathscr{I}, \mathscr{J}$ be nonzero ideals of $\Omega, \mathcal{M}$ a module of rank greater than 1. Then any monomorphism from $\mathscr{J} / \mathscr{I} \mathscr{J}$ to $\mathcal{M} / \mathscr{I} M$ can be realized by a monomorphism from $\mathscr{J}$ to $\mathcal{M}$ with pure image.

Proof. Write $\mathcal{M}$ as a direct sum

$$
\bigoplus_{i=1}^{k} \mathscr{J}_{i}
$$

of ideals. Assume by symmetry that the map $\mathscr{I} / \mathscr{I} \mathscr{I}$ to $\mathscr{I}_{1} / \mathscr{I}_{1}$ is nonzero. Choose any lifting from $\mathscr{J}$ to $\mathscr{J}_{1}$. It is nonzero, so it is a monomorphism whose image is pure except primes $P_{j}$ of torsion $\mathscr{J}_{1} / h(\mathscr{J})$. For $i>1$, require the map $f$ from $\mathscr{J}$ to $\oplus \mathscr{I}_{i}$ to lift the given mapping modulo $\mathscr{I}$ and at all primes $P_{j}$ not in $\mathscr{I}$, require it to be a monomorphism from $\mathscr{I} / P_{j} \mathscr{I}$ to $\oplus \mathscr{I}_{i} / P_{j} \mathscr{F}_{i}$. Such a monomorphism exists since the modules $\mathscr{J}_{i} / P_{j} \mathscr{I}_{i}$ and $\mathscr{I} / P_{j} \mathscr{I}$ are isomorphic and by the Chinese remainder theorem we can combine different primes. By Proposition 3.3 a lifting exists. Then at all $P_{i}$ we have pureness from the modulo $P_{i}$ monomorphism.

LEMMA 3.5. Let $\mathcal{M}, \mathcal{N}$ be isomorphic torsion free $\Omega$-modules, and $h$ an homomorphism from $\mathcal{M}$ to $\mathcal{N}$. Choose a given isomorphism $g$ from $\mathcal{M}$ to $\mathcal{N}$ and define $\operatorname{det}(h)$ as the determinant of the is vector map

$$
h g^{-1}: \mathcal{N} \otimes \boldsymbol{Q} \rightarrow \mathcal{N} \otimes \boldsymbol{Q}
$$

modulo units of $\Omega$. Then $\operatorname{det}(h)$ is an algebraic integer and is a unit if $h$ is an isomorphism. 
Proof. If $h$ is an endomorphism of $\mathcal{N}$, then the additive group of matrices spanned by $I, h, h^{2}, \ldots, h^{n}$ is a subgroup of a finite sum of copies of $Z$ namely $\operatorname{Hom}_{Z}(\mathcal{M}, \mathcal{N})$, where $I$ is an identity matrix. Therefore, some finite set is spanning by an ascending chain condition. So all higher powers of $h$ are integral sums of a finite set of powers. This implies $h$ and so all its eigenvalues satisfy a monic polynomial with coefficients in $Z$. If $h$ is an automorphism of $\mathcal{N}$ then its determinant is a unit, since the inverse is also an algebraic integer. The choice of $g$ affects det $(h)$ only by multiplying it by a unit as a consequence.

LEMMA 3.6. Let $\mathcal{M}$ be a torsion free $\Omega$-module. Define $d_{1}$ of an element of End $(\mathcal{M})$ by determinant on the vector space $\mathcal{M} \otimes Q$ and determinant $d_{2}$ on End $(\mathcal{M} / \mathscr{M})$ for a nonzero $\Omega$-ideal $\mathscr{F}$ by $\mathcal{M} / \mathscr{M}$ being a free module over $\Omega / \mathscr{I}$. Then $d_{2}$ is the modulo 9 reduction of $d_{1}$.

Proof. It suffices to verify this for $\mathscr{I}$ a power of a prime since the mapping from $\Omega / \mathscr{I}$ to $\oplus \Omega / \mathscr{P}^{n}$ over prime power factors $\mathscr{P}^{n}$ of $\mathscr{I}$ is a monomorphism. For a prime ideal $\mathscr{P}$ the module $\mathcal{M}$ over the local ring $\Omega_{\mathscr{P}}$ is free, and this determinant agrees with that over $\Omega / \mathscr{P}^{n}$ and over $\Omega \otimes Q$, since a basis over $\Omega_{\mathscr{P}}$ gives a basis over those coefficients.

\section{Reduction to filtered modules}

THEOREM 4.1. For finitely generated torsion free, nilpotent $\Omega[t]$-modules $\mathcal{M}, \mathcal{N}$ let $h_{0}$ be the composition

$$
\operatorname{Hom}(\mathcal{M}, \mathcal{N}) \rightarrow \operatorname{Hom}(\mathscr{M}, \mathcal{N} / t \mathcal{N}) \rightarrow \operatorname{Hom}(\mathscr{M} / t \mathcal{M}, \mathcal{N} / t \mathcal{N})
$$

Then Img $\left(h_{0}\right)$ lies in the subgroup $H_{f}$ of filtered homomorphisms for the filtration by $\operatorname{Ker}\left(t^{n}\right)$. Img $\left(h_{0}\right)$ is computable and can be specified by a finite system of congruences and linear equations over $\Omega \otimes Q$. An element $f$ is a near epimorphism if and only if $h_{0}(f)$ is. Given any cokernel bound and congruences in Hom $(\mathcal{M}, \mathcal{N})$ we can find a cokernel bound and congruences of $H_{f}$ such that near epimorphism exists with these data in $\operatorname{Hom}(\mathcal{M}, \mathcal{N})$ if and only if one exists with the data in $H_{f}$, which preserves filtration. Here $H_{f}$ is the set of filtration preserving homomorphisms.

(In this paper, all sets of congruences mentioned as such are finite.)

Proof. Any homomorphism must map the kernel of $t^{n}$ to the kernel of $t^{n}$. We first show that over $\Omega \otimes Q$ the image of $h_{0}$ is equal precisely to $H_{f}$. We may assume $\mathcal{M}$, $\mathcal{N}$ are in Jordan form. Express each as a sum of cyclic summands. Assume $\mathcal{M}, \mathcal{N}$ over $\Omega \otimes Q$ cyclic. Let them have bases $t^{i} v, t^{j} \omega ; i=0, \ldots, m_{1}, j=0, \ldots, n_{1}$. Then homomorphism from $\mathscr{M}$ to $\mathcal{N}$ are in 1-1 correspondence with elements

$$
\sum_{i+m_{1}+1>n_{1}} c_{i} t^{i} \omega
$$

which are the image of $v, c_{i} \in \Omega \otimes Q$. Under $h_{0}$ this maps to zero, or is onto according to whether $i=0$ may occur, i.e., $m_{1}+1 \leq n_{1}$ or $m_{1}+1>n_{1}$, i.e., $m_{1} \geq n_{1} . \operatorname{Ker}\left(t^{n}\right.$ on $\mathcal{M}) / \boldsymbol{M}$ is zero for $n \leq m_{1}$ and is spanned by $v, n>m_{1}$. $\operatorname{Ker}\left(t^{n}\right.$ on $\left.\mathcal{N}\right) / t \mathcal{N}$ is zero for $n \leq n_{1}$ is spanned by $v_{1}, n>n_{1}$. Therefore, a filtration preserving homomorphism for $m_{1}<n_{1}$ sends $\operatorname{Ker}\left(t^{m_{1}+1}\right.$ on $\left.\mathcal{M}\right) / t M$ to zero and is zero. If $m_{1} \geq n_{1}$ all homomorphisms preserve filtration. Therefore over $\Omega$ the image of $h_{0}$ in $H_{f}$ has finite index. 
The image of $h_{0}$ can be found by solving a finite system of linear equations. These amount to congruences on $H_{f}$ specifying integrality.

More generally, we can find a finite set of congruences by expressing the fact that $f$ exists satisfying any given finite set of congruences, i.e., we can determine $h_{0}(\mathfrak{S})$ for any coset $\sqrt{5}$ of a subgroup of finite index, by linear algebra. Since the cokernel of the $h_{0}(f)$ from $\mathcal{M} / t \mathcal{M}$ to $\mathcal{N} / t \mathcal{N}$ is a quotient of the cokernel of $f$ from $\mathcal{M}$ to $\mathcal{N}$, this gives a bound one way. Given a bound on the cokernel of $h_{0}(f)$ we have the same bound on the cokernel the map $f$ induces

$$
\operatorname{Hom}\left(t^{i} \mathcal{M} / t^{i+1} \mathcal{M}\right) \rightarrow \operatorname{Hom}\left(t^{i} \mathcal{N} / t^{i+1} \mathcal{N}\right)
$$

since multiplying by $t^{i}$ is an epimorphism. The product of these bounds the order of the cokernel $\mathcal{M}$ to $\mathcal{N}$.

In general, the cokernel of any map of filtered groups has its order dividing the product of the orders of the cokernels of the map on quotient groups.

If we reduce by this number any more restrictive bound on the cokernel above can be expressed by a finite system of congruences. As noted above, existence of an element satisfying these congruences in $\operatorname{Hom}(\mathcal{M}, \mathcal{N})$ can be expressed in terms of congruences on $H_{f}$. If there exists an element in $H_{f}$ with the given bound on its cokernel, satisfying these congruences, choose any lifting satisfying the congruences in Hom $(\mathcal{M}, \mathcal{N})$. This will be the required near epimorphism. And these conditions are necessary.

By restricting to various submodules of finite index of $\mathcal{N} / t \mathcal{N}$ we may assume the problem is to find an epimorphism instead of a near epimorphism of filtered $\Omega$-modules, in specified congruence classes. We consider all submodules which have an index of at most the given bound.

5. Algorithm for filtered epimorphisms

THEOREM 5.1. Let $\mathcal{M}, \mathcal{N}$ be torsion free filtered finitely generated $\Omega$-modules, filtered by $\mathcal{M}_{i}, \mathcal{N}_{i}$ :

$$
\begin{aligned}
& \mu=\mathscr{M}_{n} \supset \mathcal{M}_{n-1} \supset \cdots \supset \mathcal{M}_{1} \supset\{0\}=\mathcal{M}_{0} \\
& \mathcal{N}=\mathcal{N}_{n} \supset \mathcal{N}_{n-1} \supset \cdots \supset \mathcal{N}_{1}\{0\}=\mathcal{N}_{0}
\end{aligned}
$$

Let each $\mathcal{M}_{i}$ be a direct summand of $\mathcal{M}_{1}$ and each $\mathcal{N}_{i}$ of $\mathcal{N}$. A modulo $\mathscr{I}$ filtered epimorphism $\mathrm{g}$ can be realized integrally (i.e., $\mathcal{M}$ to $\mathcal{N}$ ) if and only if (i) the determinant of $g: \mathcal{M} / \mathcal{M}_{i} \rightarrow \mathcal{N} / \mathcal{N}_{i}$ is modulo $\mathscr{I}$ reduction of a unit whenever $\mathcal{M} / \mathcal{M}_{i}$ and $\mathcal{N} / \mathcal{N}_{i}$ are isomorphic; (ii) $\mathcal{M} / \mathcal{M}_{i}$ and $\mathcal{N} / \mathcal{N}_{i}$ are isomorphic whenever they have equal rank.

Proof. Necessity follows from Lemma 3.7, and epimorphisms of equal rank modules being isomorphisms. Observe that the map $f$ to be constructed yields epimorphisms $\mathscr{M} / \mathscr{M}_{i}$ to $\mathcal{N} / \mathcal{N}_{i}$ but need not yield epimorphims $\mathcal{M}_{j} / \mathscr{M}_{i}$ to $\mathcal{N}_{i} / \mathcal{N}_{i}$. The former condition is guaranteed rationally by modulo $\mathscr{I}$ epimorphism since modulo $\mathscr{I}$ the ranks of all $\mathscr{M}_{i}$ and $\mathscr{M} / \mathscr{M}_{i}$ equal their ranks over $\Omega \otimes Q$ since they are summands. Any module modulo $\mathscr{I}$ is isomorphic to a free module.

Assume (i), and (ii) hold. Each $\mathscr{M}_{i}$ is a pure submodule of $\mathscr{M}$ and therefore of all submodules of $\mathcal{M}$ containing $\mathcal{M}_{i}$. We split each $\mathcal{M}_{i}$ as $\mathscr{K}_{i} \oplus \mathcal{M}_{i-1}$ and then split $\mathscr{K}_{i}$ into a sum of copies of $\Omega$ and an ideal for some modules $\mathscr{K}_{i}$. 
We first construct $f$ up to $\mathscr{M}_{j}$ where $j$ is the least integer such that $\mathscr{M} / \mathscr{M}_{j} \approx \mathcal{N} / \mathcal{N}_{j}$. Let $\mathscr{K}_{i} \simeq \mathscr{K}_{i a(i)} \oplus \mathscr{K}_{i, a(i)-1} \oplus \cdots \oplus \mathscr{K}_{i 1}$ (or $\mathscr{K}_{i}=0, a(i)=0$ ) where $\mathscr{K}_{i 1}, \ldots, \mathscr{K}_{i, a(i)-1}$ are isomorphic to $\Omega, \mathscr{K}_{i a(i)}$ is isomorphic to some ideal of $\Omega$. We construct $f$ inductively from $\mathscr{K}_{i s}$ to $\mathcal{N}_{i}$ given a mapping on $\mathscr{M}_{i-1}, \mathscr{K}_{i 1}, \ldots, \mathscr{K}_{i s-1}$. We require these 5 properties to hold. (1) Given $f$ from $\mathscr{K}_{i s-1} \oplus \cdots \mathscr{K}_{i 1} \oplus \mathscr{M}_{i-1}$ into $\mathcal{N}_{i} f$ has maximum rank on $\mathscr{K}_{i s} \oplus \cdots \oplus \mathscr{K}_{i 1} \oplus \mathcal{M}_{i-1}$. (2) The torsion in $\mathcal{N} / f\left(\mathscr{K}_{i s} \oplus \cdots \oplus \mathscr{K}_{i 1} \oplus \mathcal{M}_{i-1}\right)$ is cyclic at all primes not dividing $\mathscr{I}$. (3) If $f$ is rationally onto from $\mathscr{K}_{i s} \oplus \cdots \oplus \mathscr{K}_{i 1} \oplus \mathcal{M}_{i-1}$ into $\mathcal{N}_{i}$ then the cokernel of $f$ from $\mathscr{K}_{i s} \oplus \cdots \oplus \mathscr{K}_{i 1} \oplus \mathcal{M}_{i-1}$ into $\mathcal{N}_{i}$, if $a(i) \neq 0$ has torsion only primes dividing $\mathscr{I}$. (4) Modulo $\mathscr{I}, f$ equals $g$. (5) If $\operatorname{Rank}\left(\mathscr{M} / \mathscr{M}_{i}\right)=\operatorname{Rank}\left(\mathcal{N} / \mathcal{N}_{i}\right)$ then $f$ is an epimorphism $\mathcal{M}_{i}$ to $\mathcal{N}_{i}$.

The first condition can be restated either as $f$ is onto from $\mathscr{K}_{i s-1} \oplus \cdots \oplus \mathscr{K}_{i} \oplus \mathcal{M}_{i-1}$ into $\mathcal{N}_{i}$ rationally or $f$ is $1-1$ on $\mathscr{K}_{i s}$ and $f\left(\mathscr{K}_{i s}\right) \cap f\left(\mathscr{K}_{i s-1} \oplus \cdots \oplus \mathscr{K}_{i 1} \oplus \mathcal{M}_{i-1}\right)=\{0\}$. To guarantee the second condition it will suffice to consider the latter case, since otherwise the torsion will decrease.

Let $\mathscr{P}$ be the pure submodule of $\mathcal{N}_{i}$ generated by $f\left(\mathscr{K}_{i s-1} \oplus \cdots \oplus \mathscr{K}_{i 1} \oplus \mathcal{M}_{i-1}\right)$. Then the second condition will follow if we map $\mathscr{K}_{i s}$ into $\mathcal{N}_{i} / \mathscr{P}$ so that its cokernel has torsion relatively prime to that of $\mathcal{N}_{i} / f\left(\mathscr{K}_{i s-1} \oplus \cdots \oplus \mathscr{K}_{i 1} \oplus \mathcal{M}_{i-1}\right)$ except at the prime $\mathscr{I}$. Conditions (1), (2) will follow by choosing a map on $\mathscr{K}_{i s}$ in a certain congruence class modulo an ideal $\mathscr{I}_{1}$ corresponding to torsion in $\mathcal{N}_{i} / f\left(\mathscr{K}_{i s-1} \oplus \cdots \oplus \mathscr{K}_{i 1} \oplus \mathcal{M}_{i-1}\right)$ at primes not dividing $\mathscr{I}$, and modulo some other prime ideal $\mathscr{P}_{1}$. The former can be chosen to guarantee that $N_{i} /\left(\mathscr{P}+f\left(\mathscr{K}_{i s}\right)\right)$ has no torsion at primes of $\mathscr{I}_{1}$ and the latter to guarantee that $f\left(\mathscr{K}_{\text {is }}\right)$ is rationally independent from $f\left(\mathscr{K}_{i s-1} \oplus \cdots \oplus \mathscr{K}_{i 1} \oplus\right.$ $\left.\mathscr{M}_{i-1}\right)$. These can be made compatible with (4) at the prime $\mathscr{I}$ by the Chinese remainder theorem and Proposition 3.3.

Moreover, we can guarantee (4) and (3) in the case that $f$ previously constructed is rationally onto. First solve (3) separately by mapping the ideal $\mathscr{K}_{\text {is }}$ onto the summand of $\mathcal{N}_{i} / f\left(\mathscr{K}_{i s-1} \oplus \cdots \oplus \mathscr{K}_{i 1} \oplus \mathcal{M}_{i-1}\right)$ at primes not dividing $\mathscr{I}$. Since this module is cyclic and $\mathscr{K}_{\text {is }}$ modulo $\mathscr{I}_{1}$ is $\Omega$ modulo $\mathscr{I}_{1}$ we can do this. Now take the congruence class of this map modulo $\mathscr{I}_{1}$ and the congruence class at $\mathscr{I}$ of $g$ and realize them by some map by Proposition 3.3. Here $\Phi_{1}$ is as above.

If $a(i)=0$, then the previously constructed $f$ fulfills all conditions by induction. Since $\mathcal{N}_{i}$ is a pure submodule and summand of $\mathcal{N}$ no new torsion will be introduced in the cokernel by taking it into $\mathcal{N}_{i}$ instead of $\mathcal{N}_{i-1}$. If $\operatorname{Rank}\left(\mathcal{M} / \mathcal{M}_{i}\right)=\operatorname{Rank}\left(\mathcal{N} / \mathcal{N}_{i}\right)$ and $a(i)=0$, so $\mathscr{M}_{i}=\mathscr{M}_{i-1}$ then the inequality $\operatorname{Rank}\left(\mathscr{M} / \mathcal{M}_{i-1}\right) \geq \operatorname{Rank}\left(\mathcal{N} / \mathcal{N}_{i-1}\right)$ and $\mathcal{N}_{i-1} \subset \mathcal{N}_{i}$ imply $\operatorname{Rank}\left(\mathcal{M}_{i} / \mathscr{M}_{i-1}\right)=\operatorname{Rank}\left(\mathcal{N}_{i} / \mathcal{N}_{i-1}\right)$. More generally, next we deal with (5), that is, the case when $\operatorname{Rank}\left(\mathscr{M} / \mathcal{M}_{i}\right)=\operatorname{Rank}\left(\mathcal{N} / \mathcal{N}_{i}\right)$. Then $s=a(i)$. There are 2 subcases, according to whether $f: \mathscr{K}_{i s} \oplus \cdots \oplus \mathscr{K}_{i 1} \oplus \mathcal{M}_{i-1} \rightarrow \mathcal{N}_{i}$ is rationally onto or not. If it is, the procedure above yields a map which is onto except possibility at primes dividing $\mathscr{I}$ by (4). But at primes dividing $\mathscr{I}$ the modulo $\mathscr{I}$ reduction is onto. Therefore, the cokernel cannot have torsion either dividing $\mathscr{I}$ or not dividing $\mathscr{I}$. So (5) holds, We show the second subcase is impossible. It will suffice to show that the kernel of $f$ rationally from $\mathcal{M}_{i-1}$ to $\mathcal{N}_{i-1}$ has rank less than $\operatorname{Rank}\left(\mathcal{M}_{i}\right)-$ Rank $\left(\mathcal{N}_{i}\right)$ since in that case the kernel will have to increase, and since our construction maximizes rank as we extend when we have defined the map on $\mathscr{K}_{i s-1}$ 
it must be rationally onto, and the map on $\mathscr{K}_{i s}$ will be rationally in the previous image.

Modulo $\Phi$ we have an isomorphism $\mathscr{M} / \mathcal{M}_{i} \rightarrow \mathcal{N} / \mathcal{N}_{i}$ and an epimorphism which is not an isomorphism $\mathscr{M} / \mathcal{M}_{j} \rightarrow \mathcal{N} / \mathcal{N}_{j}, j<i$. All submodules $\mathcal{M}_{i}, \mathcal{N}_{i}$ are pure and rational ranks equal the ranks of modulo $\mathscr{I}$ reductions. These facts also imply a modulo $\mathscr{I}$ epimorphism $\mathcal{M}_{i} \rightarrow \mathcal{N}_{i}$. Thus; (1) Rank $\left(\mathcal{M}_{i}\right)-\operatorname{Rank}\left(\mathcal{M}_{j}\right)>\operatorname{Rank}\left(\mathcal{N}_{i}\right)-$ Rank $\left(\mathcal{N}_{j}\right)$ since we have an epimorphism of quotient groups which is not isomorphism.

We assert that the kernel of $f$ on $\mathcal{M}_{k}$ rationally for $k<i$ has rank

(2) $\max _{j \leq k}\left\{0, \operatorname{Rank}\left(\mathcal{M}_{j}\right)-\operatorname{Rank}\left(\mathcal{N}_{j}\right)\right\}$.

This is a lower bound, since the rank of the kernel $\mathscr{M}_{j}$ to $\mathcal{N}_{j}$ is at least Rank $\left(\mathcal{M}_{j}\right)-$ $\operatorname{Rank}\left(\mathcal{N}_{j}\right)$. For $j=0$, this gives zero; for $j=1$, zero or $\operatorname{Rank}\left(\mathcal{M}_{1}\right)-\operatorname{Rank}\left(\mathcal{N}_{1}\right)$ which is correct as we have a rational epimorphism $\mathscr{M}_{1}$ to $\mathcal{N}_{1}$ if $\operatorname{Rank}\left(\mathscr{M}_{1}\right) \geq \operatorname{Rank}\left(\mathcal{N}_{1}\right)$, by construction.

Let the kernel have rank $r_{0}$ from $\mathscr{M}_{j-1}$ to $\mathcal{N}_{j-1}$. If

(3) $\operatorname{Rank}\left(\mathcal{M}_{j}\right)-\operatorname{Rank}\left(\mathcal{M}_{j-1}\right) \leq \operatorname{Rank}\left(\mathcal{N}_{j}\right)-\operatorname{Rank}\left(f\left(\mathcal{M}_{j-1}\right)\right)$

then we have a isomorphism on $\mathscr{K}_{j}$ modulo $f\left(\mathcal{M}_{j-1}\right)$ by construction. Therefore, the kernel on $\mathcal{M}_{j}$ has rank $r_{0}$. In other case, we have an epimorphism and the kernel on $\mathcal{M}_{j}$ has rank equal to Rank $\left(\mathcal{M}_{j}\right)-\operatorname{rank}\left(\mathcal{N}_{j}\right)$.

Condition (3) can be restated:

$$
\begin{aligned}
& \operatorname{Rank}\left(\mathcal{M}_{j}\right)-\operatorname{Rank}\left(\mathcal{M}_{j-1}\right) \leq \operatorname{Rank}\left(\mathcal{N}_{j}\right)-\operatorname{Rank}\left(\mathcal{M}_{j-1}\right)+r_{0}, \\
& \operatorname{Rank}\left(\mathcal{M}_{j}\right)-\operatorname{Rank}\left(\mathcal{N}_{j}\right) \leq r_{0} .
\end{aligned}
$$

This gives an induction establishing formula (2) above. We have $\operatorname{Rank}\left(\mathcal{M}_{i}\right)-$ $\operatorname{Rank}\left(\mathcal{N}_{i}\right)>\operatorname{Rank}\left(\mathcal{M}_{j}\right)-\operatorname{Rank}\left(\mathcal{N}_{j}\right)$ by $(1)$. We have $\operatorname{Rank}\left(\mathcal{M}_{i}\right)-\operatorname{Rank}\left(\mathcal{N}_{i}\right)>0$ because otherwise the modulo $\mathscr{I}$ epimorphism $g$ from $\mathcal{M}_{i}$ to $\mathcal{N}_{i}$ gives a modulo $\mathscr{I}$ isomorphism and so a modulo $\mathscr{I}$ isomorphism $\mathcal{M}$ to $\mathcal{N}$ contrary to assumption. Therefore, Rank $\left(\mathscr{M}_{i}\right)-\operatorname{Rank}\left(\mathcal{N}_{i}\right)$ exceeds the value of formula (2). This concludes the proof that the second case cannot occur.

Now we have extended the map to $\mathcal{M}_{s}$ such that $\mathscr{M} / \mathcal{M}_{s} \simeq \mathcal{N} / \mathcal{N}_{s}$. It suffices to solve the problem of finding an isomorphism on the quotient groups

$$
\mathscr{M} / \mathcal{M}_{s} \simeq \mathcal{N} / \mathcal{N}_{s}
$$

equal to $g$ modulo $\mathscr{I}$. For if we have such a map, and a map $\mathcal{M}_{s}$ to $\mathcal{N}_{s}$ we can obtain an arbitrary $g: \mathcal{M} \rightarrow \mathcal{N}$ modulo $\mathscr{I}$ by adding a map $\mathscr{M} / \mathcal{M}_{s} \rightarrow \mathcal{N}_{s}$ which need only be a homomorphism agreeing with $g$ modulo $\mathscr{I}$. By Proposition 3.3 this homomorphism will exist. The isomorphism and epimorphism on quotient groups imply the total map is an epimorphism.

By the same reasoning it suffices to construct isomorphism

$$
\mathcal{M}_{s(i)} / \mathscr{M}_{s(i-1)} \rightarrow \mathcal{N}_{s(i)} / \mathcal{N}_{s(i-1)},
$$

where $s(r)=n>s(r-1)>\cdots>s(1)=s$ and

$$
\operatorname{Rank}\left(\mathcal{M} / \mathscr{M}_{s(i)}\right) \simeq \operatorname{Rank}\left(\mathcal{N} / \mathcal{N}_{s(i)}\right)
$$

and the sequence $s(i)$ cannot be further refined. As in the last paragraph the difference 
between such maps on the direct sum

$$
\bigoplus \mathscr{M}_{s(i)} / \mathscr{M}_{s(i-1)}
$$

and $g$ modulo $\mathscr{I}$ on $\mathscr{M} / \mathcal{M}_{s}$ can be made zero by adding homomorphisms in turn from $\mathcal{M}_{s(i)} / \mathcal{M}_{s(i-1)}$ to $\mathcal{N}_{s(i-1)}$.

So we have reduced the problem to the case $\operatorname{Rank}(\mathscr{M})=\operatorname{Rank}(\mathcal{N})$, and for no $s$ greater than zero and less than $n$ is

$$
\operatorname{Rank}\left(\mathcal{M} / \mathscr{M}_{s}\right)=\operatorname{Rank}\left(\mathcal{N} / \mathcal{N}_{s}\right)
$$

We define $\mathscr{K}_{\text {is }}$ as before and construct $f$ on them in turn. We require $\left(1^{\prime}\right) f$ is rationally $1-1$. ( $\left.2^{\prime}\right) f$ has pure image. $\left(3^{\prime}\right) f$ modulo $\mathscr{I}$ equals $g$, at each stage.

First suppose $\mathscr{K}_{i s}$ is not $\mathscr{K}_{n a(n)}$, the final ideal. We claim the cokernel of $f$ from $\mathscr{K}_{i, s-1} \oplus \cdots \oplus \mathscr{K}_{i 1} \oplus \mathcal{M}_{i-1}$ into $\mathcal{N}_{i}$ has rank at least 2 . If not then Rank $\left(\mathcal{M}_{i}\right) \geq \operatorname{Rank}\left(\mathcal{N}_{i}\right)$ and with $\operatorname{Rank}\left(\mathscr{M} / \mathscr{M}_{i}\right) \geq \operatorname{Rank}\left(\mathcal{N} / \mathcal{N}_{i}\right)$ we have $\operatorname{Rank}\left(\mathcal{M}_{i}\right)=\operatorname{Rank}\left(\mathcal{N}_{i}\right)$ but we assumed this did not happen.

If the cokernel has rank at least 2 it is a direct summand since the image is pure. Define $f_{1}$ into the direct summand $C$, where $C \oplus f\left(\mathscr{K}_{i s-1} \oplus \cdots \oplus \mathscr{M}_{i-1}\right)=\mathcal{N}_{i}$ by Proposition 3.4 so as to lift $g$ modulo $f\left(\mathscr{K}_{i s-1} \oplus \cdots \oplus \mathcal{M}_{i-1}\right)$ and to have pure image. Define $f_{2}$ from $\mathscr{K}_{i s}$ into $f\left(\mathscr{K}_{i s-1} \oplus \cdots \oplus \mathcal{M}_{i-1}\right)$ and to have pure image. Define $f_{2}$ from $\mathscr{K}_{\text {is }}$ into $f\left(\mathscr{K}_{i s-1} \oplus \cdots \oplus \mathscr{M}_{i-1}\right)$ by Proposition 3.3 so that $f_{2}$ lifts $g-\left(f_{1}(\bmod \mathscr{I})\right)$. Then $f=f_{1}+f_{2}$ satisfies $\left(1^{\prime}\right),\left(2^{\prime}\right),\left(3^{\prime}\right)$. Now suppose $\mathscr{K}_{i s}$ is $\mathscr{K}_{n \alpha(n)}$, the final ideal. Then the cokernel must have rank 1. Define $f$ from $\mathscr{K}_{\text {is }}$ into the previous image again by Proposition 3.3 to be a homomorphism lifting $g$. The cokernel is a single idea. By the cancellation Theorem 3.2 and the Assumption (ii) of this theorem, it is isomorphic to $\mathscr{K}_{i s}$. Isomorphisms from the cokernel to $\mathscr{K}_{i s}$ are in 1-1 correspondence with units of $\Omega$, acting by multiplication to preserve an ideal of $\Omega$, and homomorphisms in 1-1 correspondence with elements of $\Omega$. For any homomorphism $f$ extending $f$ on the kernel and lifting $g$, the determinant of $f$ is the determinant, locally at each prime, of a block triangular matrix

$$
\left[\begin{array}{cc}
f_{c} & 0 \\
* & f_{i}
\end{array}\right],
$$

where $f_{c}$ is the map $\mathscr{K}_{i s}$ into the cokernel and $f_{i}$ is the isomorphism into the image previously defined. So the determinant is

$$
f_{c}\left(\operatorname{Det}\left(f_{i}\right)\right) \text {. }
$$

Det $\left(f_{i}\right)$ is a unit since $f_{i}$ is an isomorphism. Let $f_{c}$ be a unit such that modulo $\mathscr{I}$,

$$
\left(\operatorname{Det}\left(f_{c}\right)\right)\left(\operatorname{Det}\left(f_{i}\right)\right)=\operatorname{Det}(g) \text {, }
$$

which is a unit by Assumption (i) of this theorem. (Observe that if $\mathcal{M} / \mathcal{M}_{i}, \mathcal{N} / \mathcal{N}_{i}$ have equal ranks and $\mathscr{M} / \mathscr{M}_{j}, \mathcal{N} / \mathcal{N}_{j}$ have equal equal ranks, $i>j$ then $\mathcal{M}_{j} / \mathcal{M}_{i}, \mathcal{N}_{j} / \mathcal{N}_{i}$ have equal ranks and Assumption (ii) implies that also on them $g$ is the modulo $\mathscr{I}$ reduction of a unit.) In defining the determinants we assume the module isomorphism extends $f$ where it was previously defined. Then $f_{c}$ equals $g$ modulo $\mathscr{I}$ from $\mathscr{K}_{\text {is }}$ to the cokernel since $g$ also satisfies the above determinant equation, since $f_{c}$ is multiplication by a unit it is an isomorphism. 
LEMMA 5.2. A map $\mu \rightarrow \mathcal{N}$ of $\Omega$-modules is an epimorphism if and only if it is an epimorphism modulo torsion and an epimorphism modulo $m$ where $m=\mid$ Torsion $(\mathcal{N}) \mid$. Given elements $a_{1}$ of $\operatorname{Hom}\left(\mathcal{M}, \mathcal{N} /\right.$ torsion) and $a_{2}$ of $\operatorname{Hom}(\mathcal{M}, \mathcal{N} / m \mathcal{N})$ whose images under the natural maps to Hom $(\mathcal{M}, \mathcal{N} /(m \mathcal{N}+$ torsion $))$ are equal, there exists a unique element a of $\operatorname{Hom}(\mu, \mathcal{N})$ yielding $a_{1}, a_{2}$ under the natural maps.

Proof. To prove existence, let $T=$ Torsion $(\mathcal{N})$. Let $\mathcal{N}=T \oplus E$. Then

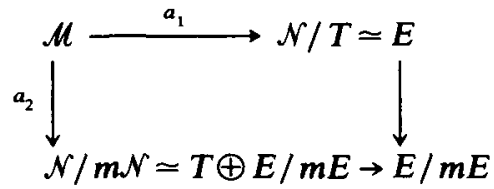

commutes by hypothesis. Let $a_{2}(x)=\alpha(x)+\beta(x)$ where $\alpha$ is from $\mu$ to $T, \beta$ is from $\mathcal{M}$ to $E / m E$. Then $a(x)=\alpha(x)+a_{1}(x)$ is a lifting.

Uniqueness holds since a kernel element would have to be zero modulo torsion and zero modulo $m$. If the cokernel of a map is zero modulo $m$, it must be torsion relatively prime to $m$, so the image contains $k \mathcal{N},(k, m)=1$. But it contains $m \mathcal{N}$ if modulo torsion we have an epimorphism, so it contains $\mathcal{N}$.

THEOREM 5.3. If $\mathcal{M}, \mathcal{N}$ are any filtered $\Omega$-modules, there is an algorithm giving all modulo $\Phi$ classes of filtered epimorphisms $\mathcal{M}$ to $\mathcal{N}$.

Proof. First, let $\mu, \mathcal{N}$ be torsion free. Let $\mathcal{M}_{i}, \mathcal{N}_{i}$ be the given filtration. Let $\mathscr{T}_{i}=\left(M_{i} \otimes Q\right) \cap \mathcal{M}, \mathscr{U}_{i}=\left(\mathcal{N}_{i} \otimes Q\right) \cap \mathcal{N}$. Then any filtered map sends $\mathscr{T}_{i}$ to $\mathscr{U}_{i}$. We can decide all congruence classes of epimorphisms of the latter system by Theorem 5.1. A finite set of additional congruences gives the condition $\mathcal{M}_{i}$ to $\mathcal{N}_{i}$. Let $\mathscr{M}, \mathcal{N}$ have torsion. We must have an epimorphism

$$
\mu / \text { torsion } \rightarrow \mathcal{N} / \text { torsion }
$$

and the congruence classes of these can be decided modulo any nonzero ideal of $\Omega$.

Filtration by the images of $\mathcal{M}_{i}, \mathcal{N}_{i}$ will be preserved. There are a finite set of possibilities for maps $\mathscr{M} \rightarrow \mathcal{N} / m \mathcal{M}$ and these by Lemma 5.2 together with the map modulo torsion determine the map $\mathcal{M} \rightarrow \mathcal{N}$. From the two we can determine congruence classes of epimorphisms but there is still a question of preserving filtration.

We can express filtration preservation by saying that $\boldsymbol{M}_{i} \rightarrow \mathcal{N} \rightarrow \mathcal{N} / \mathcal{N}_{i}$ is zero. Over $Q$ this map will be zero if modulo torsion, filtration is preserved. The torsion portion has some definite exponent, the order of Torsion $\left(\mathcal{N} / \mathcal{N}_{i}\right), m$ and it suffices that the map be zero modulo $m$. This amounts to a finite system of congruences.

COROLlaRy 1. It is decidable if an epimorphism exists between finitely additively generated $Z[t]$-modules.

COROLLARY 2. Epimorphisms of dimension groups are decidable for primitive matrices over $\boldsymbol{Z}$.

COROllary 3. Existence of isomorphisms of dimension groups are decidable for primitive matrices over $\boldsymbol{Z}$.

Proof. For matrices with an equal number of nonzero eigenvalues any rational epimorphism of dimension groups will be monic. 


\section{Conclusion}

We can decide the existence of epimorphisms of $\mathcal{Z}(t)$-modules by splitting into problems at each eigenvalue with specified congruences. This involves near epimorphisms of nilpotent modules over an algebraic number ring, and we reduce this to a question of filtered epimorphisms of modules over the algebraic number ring. In the case when the modules are torsion free and the filtration submodules are pure we are able to obtain explicit necessary and sufficient conditions.

\section{Acknowledgment}

We would like to express our appreciation to the referee for very helpful suggestions.

\section{REFERENCES}

[1] M. Boyle, B. Marcus \& P. Trow. Resolving maps and the dimension group for shifts of finite type. Memoirs of the Amer. Math. Soc. (Amer. Math. Soc., Providence, RI, 1987).

[2] Z. I. Borevich \& I. R. Shafarevich. Zahlentheorie. Birkhauser: Basel, 1966.

[3] H. Cartan \& S. Eilenberg. Homological Algebra. Princeton University Press: Princeton, 1956.

[4] F. Grunewald. Solution of conjugacy problems in certain arithmetic groups. In S. I. Adian, W. W. Boone, G. Higman, eds., Word Problems II, pp. 101-139. North-Holland: Amsterdam, 1980.

[5] F. Grunewald \& D. Segal. Some general algorithms. I. Arithmetic groups, Ann. Math. 112 (1980), 531-583.

[6] F. Grunewald \& D. Segal. Decision problems concerning S-arithmetic groups. J. Symp. Logic 50 (1985), 734-772.

[7] K. H. Kim \& F. W. Roush. Some results on decidability of shift equivalence. J. Comb., Inf. \& Sys. Sci. 4 (1979), 123-146.

[8] K. H. Kim \& F. W. Roush. Decidability of shift equivalence. Dynamical Systems. ed., J. C. Alexander. Lecture Notes in Mathematics 1342 (Springer Verlag, Berlin, 1988).

[9] K. H. Kim \& F. W. Roush. Undecidability of module homomorphisms. Proc. Amer. Math. Soc., 104 (1988), 374-376.

[10] J. Serre, Modules projectifs et espaces fibrés à fibre vectorielle. Algebra et Théorie des Nombres. Seminaire Dubreil: Paris, 1958. 